\title{
18
}

\section{Characterization of Green Roof Stormwater Runoff Quality}

\section{Jieyun Chen and James Li}

Green roofs are recognized as effective means of stormwater quantity control through runoff volume reduction and peak discharge attenuation. A properly designed and constructed green roof can improve stormwater quality by reducing the pollutant loads to receiving waters. In this study, with onsite data monitored at the rooftop, green roof stormwater was analyzed from the perspectives of both runoff quantity and runoff quality in an attempt to interpret the basic relationship between rainfall and runoff, and to characterize pollutant loads along with event mean concentrations (EMCs). From correlation analysis of a total of twelve major pollutants found in green roof runoff, it is seen that the correlation between the loads of two pollutants appears to be stronger than the correlation between the EMCs of the two pollutants. As one of the common pollutants is suspended solids, the correlations between suspended solids and other pollutants were evaluated for the possibility of using the loads of suspended solids as a surrogate to predict other pollutant loads. Further statistical analysis of the cumulative density functions (CDFs) of pollutant loads reveals that the log-normal distribution appears to fit the observed data reasonably well when compared with the CDFs of normal and exponential distributions. The normal distribution tends to significantly overestimate the CDFs of pollutant loads. From the extrapolation of the exceedance probability of pollutant loads based on statistics of the sample data, it is seen that the log-Pearson distribution is capable of providing estimates of pollutant loads which are close to the estimates from the log-normal distribution for a given return period. In comparison, the Pearson distribution may significantly underestimate pollutant loads with reference to the estimates of the log-normal or log-Pearson distributions.

Chen, J. and J. Li. 2011. "Characterization of Green Roof Stormwater Runoff Quality." Journal of Water Management Modeling R241-18. doi: 10.14796/JWMM.R241-18.

(C) CHI 2011 www.chijournal.org ISSN: 2292-6062 (Formerly in Cognitive Modeling of Urban Water Systems. ISBN: 978-0-9808853-4-7) 


\subsection{Introduction}

As a result of increasing public awareness of sustainable development, there is a growing interest in the planning and design of green roofs for sustainable infrastructure. In 2009 the City of Toronto passed a new green roof bylaw which consists of a green roof construction standard and a mandatory requirement for green roofs on all classes of new buildings. The bylaw requires up to $50 \%$ green roof coverage on multi-unit residential dwellings over six storeys, and on commercial and industrial buildings. Moreover, other larger residential projects require greater green roof coverage, ranging from $20 \%$ to $50 \%$ of the roof area. In general, green roofs may be constructed with various media and vegetation covers, but in practice there are two types of green roofs: intensive and extensive. Compared with intensive green roofs, extensive green roofs typically have shallow media depths $(<150 \mathrm{~mm})$ with vegetation that requires minimal irrigation and maintenance.

The benefits of green roofs are numerous. From the perspective of stormwater quantity control, green roofs are generally recognized as an effective means for runoff volume reduction and peak discharge attenuation. According to the study by Bliss et al. (2009), a green roof reduces runoff volume by $\leq 70 \%$ and peak discharge rate by $6 \%$ to $70 \%$ of the control roof rates. From the perspective of stormwater quality, studies show that as much as $95 \%$ of pollutants such as cadmium, copper and lead, and $16 \%$ of zinc have been taken out of rainwater by green roof systems (Johnston and Newton, 1996). The water quality of green roof stormwater runoff varies depending on the characteristics of the runoff and the pollutants accumulated on the roof (Teemusk and Mander, 2007). Seters et al. (2009) found that loads of most chemical variables in green roof runoff were lower than those from conventional roofs. A study by the U.S. Environmental Protection Agency (USEPA, 2009) revealed that while a green roof may contribute more nutrients and other ions to the roof runoff, due mostly to the use of media and initial composting to provide nutrients for plants, total nutrient loading was sometimes lower, even when pollutant concentration was higher, since the total runoff from green roofs was about $50 \%$ less than the runoff from flat asphalt roofs.

In the past, various studies were performed to characterize general urban stormwater runoff (McLeod et al., 2006; Lee and Bang, 2000). Specific studies were also conducted to investigate stormwater runoff quality from highway or urban roadways (Flint and Davis, 2007; Keiko and Shigeo, 2006). This present study seeks a better understanding of green roof runoff, and statistical analysis is performed to characterize green roof stormwater quality based on correlation analysis and probability distributions. Among 
the commonly used cumulative distribution functions, namely normal, lognormal and exponential distributions, the results from this study indicate that the log-normal distribution function fits pollutant loads reasonably well.

The study site with the constructed extensive green roof is located on the rooftop of the York University (Toronto) computer science building. The rooftop of the building is covered by two surfaces: shingles (control roof) with an area of $131 \mathrm{~m}^{2}$, and the garden with an area of $241 \mathrm{~m}^{2}$ and a slope of $10 \%$. The extensive green roof consists of a $140 \mathrm{~mm}$ substrate and is vegetated with wildflowers. The growing medium is composed of crushed volcanic rock, compost, blonde peat, cooked clay and washed sand. Stormwater runoff from the building drains to Black Creek, one of the tributaries of the Humber River, which empties into Lake Ontario. More detailed description of the structure of the extensive green roof including equipment setup and data monitoring can be found in the previous study (TRCA, 2006).

\subsection{Correlation Analysis}

\subsubsection{Pollutant Event Mean Concentrations}

For the purpose of characterization of green roof stormwater runoff, water quality sampling was taken for a total of 21 events during the 2003 and 2004 monitoring seasons (water quality was not monitored in 2005 and more recent monitoring data was not available for this study). These water quality samples were submitted to the Ontario Ministry of the Environment laboratories for analysis of general chemistry (suspended solids, chloride), nutrients (nitrogen and phosphorus compounds), bacteria, metals, and polycyclic aromatic hydrocarbons. The volume weighted mean concentration (VWMC) for each water quality variable was estimated as:

$$
V W M C=\sum_{i=1}^{n} V_{i} \cdot E M C_{i} / \sum_{i=1}^{n} V_{i}
$$

where:

$$
\begin{aligned}
V_{i}= & \text { total volume of runoff measured for a given event } i, \\
E M C_{i}= & \text { event mean concentration of the variable for a given } \\
& \text { event } i\left(\mathrm{~g} / \mathrm{m}^{3}\right), \text { and } \\
n= & \text { number of events sampled. }
\end{aligned}
$$

The pollutant event mean concentrations (EMCs) for twelve water quality parameters in green roof stormwater runoff were analyzed in this study. Based on a total of 18 sample measurements, a summary of the general statistics for green roof pollutant EMCs is given in Table 18.1. 
Table 18.1 General statistics for green roof pollutant EMCs.

\begin{tabular}{lccccc}
\hline \multicolumn{1}{c}{ Pollutant } & Range & Guideline* & Mean & Standard deviation & Skewness \\
\hline $\mathrm{SS}(\mathrm{mg} / \mathrm{L})$ & $1.25-4.3$ & & 2.01 & 1.14 & 0.988 \\
$\mathrm{BOD}(\mathrm{mg} / \mathrm{L})$ & $0.5-3.7$ & & 1.93 & 0.95 & 0.478 \\
$\mathrm{COD}(\mathrm{mg} / \mathrm{L})$ & $31.0-250.0$ & & 84.06 & 56.90 & 1.561 \\
$\mathrm{TP}(\mathrm{mg} / \mathrm{L})$ & $0.1-0.9$ & & 0.36 & 0.25 & 0.625 \\
$\mathrm{TKN}(\mathrm{mg} / \mathrm{L})$ & $0.3-2.4$ & & 1.26 & 0.66 & 0.893 \\
$\mathrm{Fe}(\mu \mathrm{g} / \mathrm{L})$ & $9.2-56.4$ & 300 & 24.66 & 14.00 & 0.329 \\
$\mathrm{Cu}(\mu \mathrm{g} / \mathrm{L})$ & $9.5-119.0$ & 5 & 55.68 & 32.86 & 0.120 \\
$\mathrm{Al}(\mu \mathrm{g} / \mathrm{L})$ & $33.2-89.4$ & 75 & 44.90 & 16.75 & -0.069 \\
$\mathrm{Mn}(\mu \mathrm{g} / \mathrm{L})$ & $0.6-2.6$ & & 1.39 & 0.66 & -0.049 \\
$\mathrm{Ni}(\mu \mathrm{g} / \mathrm{L})$ & $0.7-3.7$ & 25 & 1.31 & 0.90 & 1.032 \\
$\mathrm{Zn}(\mu \mathrm{g} / \mathrm{L})$ & $2.1-12.9$ & 20 & 6.93 & 3.68 & -0.148 \\
$\mathrm{~Pb}(\mu \mathrm{g} / \mathrm{L})$ & $2.5-11.5$ & 5 & 3.51 & 2.60 & 1.990 \\
\hline$* \mathrm{C} / \mathrm{L}$ &
\end{tabular}

*Guidelines listed are provincial water quality objectives where available. For parameters with no PWQO, the Canadian water quality guideline is used.

The values in the table indicate that the green roof runoff has lower EMCs for suspended solids and biochemical oxygen demand (BOD) than are given in the Canadian Environmental Quality Guidelines (Canadian Council of Ministers of the Environment, 1999). However, the green roof runoff was also found to have higher EMCs for a number of pollutants including chemical oxygen demand (COD), mainly due to the garden growing media itself, none of which is a concern in terms of the receiving water guidelines. Copper was the only variable in green roof runoff with mean concentrations that exceeded receiving water guidelines. Elevated phosphorus levels in green roof runoff are a significant concern in terms of the receiving water guidelines.

In order to determine the degree of dependence of the EMCs on rainfall volume and intensity, correlations between the EMCs and rainfall intensities were calculated. These results indicate that all the correlation coefficients are generally $<0.45$. Several pollutants were found to be negatively correlated with corresponding rainfall intensities. In terms of the calculated correlation coefficients for various pollutants, it was concluded that the EMCs are not apparently dependent on rainfall intensities. In other words, the change in EMC of the pollutant is not sensitive to the variation of rainfall intensity. For instance, Figure 18.1 illustrates the correlation between the EMC of BOD and rainfall intensity. From the figure, it appears that the relationship between the EMC of BOD and rainfall intensity is not clearly defined.

Following similar procedures, correlations between the EMCs and rainfall volumes were also analyzed. Several pollutants were found negatively correlated with rainfall volume. Among the calculated results for correlation coefficients, the highest value of 0.538 is for the correlation between the EMC of Total Kjeldahl Nitrogen (TKN) and rainfall volume. A comparison 
of the calculated correlation coefficients for various pollutants indicates that the EMCs are not highly dependent on rainfall volumes. As a comparison to the correlation between the EMC of BOD and rainfall intensity shown in Figure 18.1, the correlation between the EMC of BOD and rainfall volume is illustrated in Figure 18.2. There are no apparent patterns observed in the two figures.

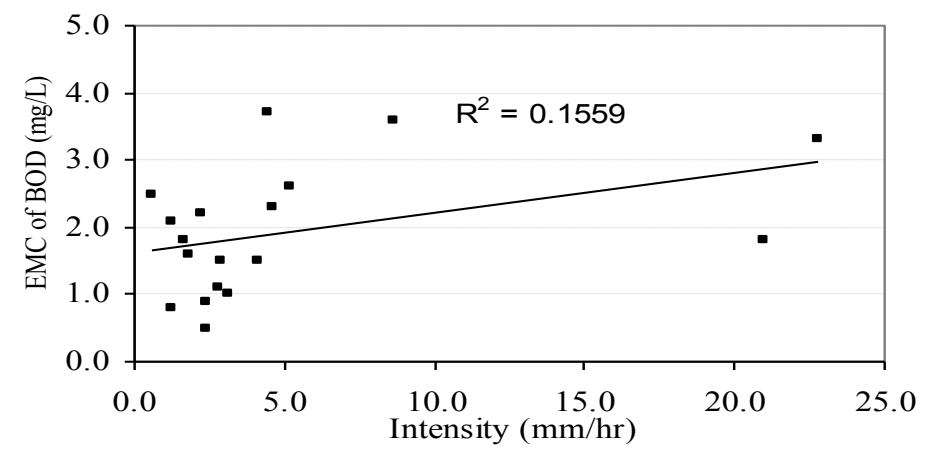

Figure 18.1 Correlation between the EMC of BOD and rainfall intensity.

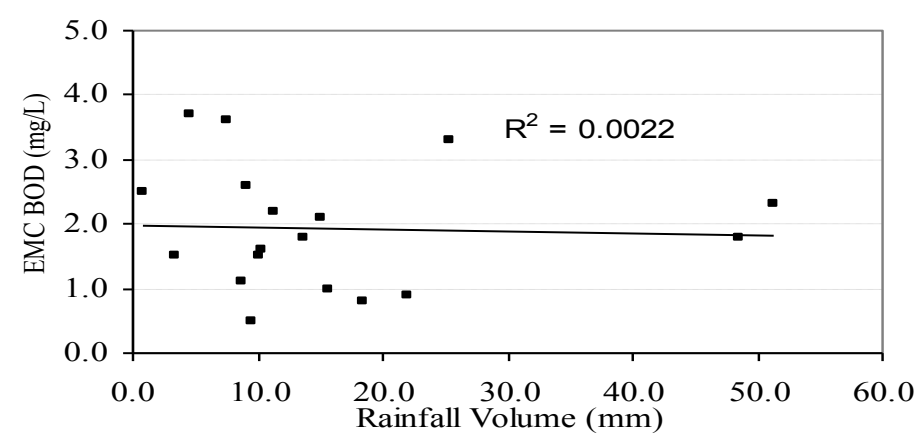

Figure 18.2 Correlation between the EMC of BOD and rainfall volume.

As a comparison with the correlations between the EMCs and rainfall volumes and intensities, correlations between the EMCs and runoff volumes were also evaluated. Among the correlation coefficients for different pollutants, it was found that the EMCs were not strongly correlated with runoff volumes. While the correlation coefficient is 0.76 for total phosphorus (TP), 0.638 for iron and 0.575 for $\mathrm{TKN}$, the correlation coefficients for other pollutants are generally $<0.5$. Several pollutants were also found to be negatively correlated with runoff volumes. For illustration, Figure 18.3 shows the correlation between the EMCs of BOD and runoff volumes. 


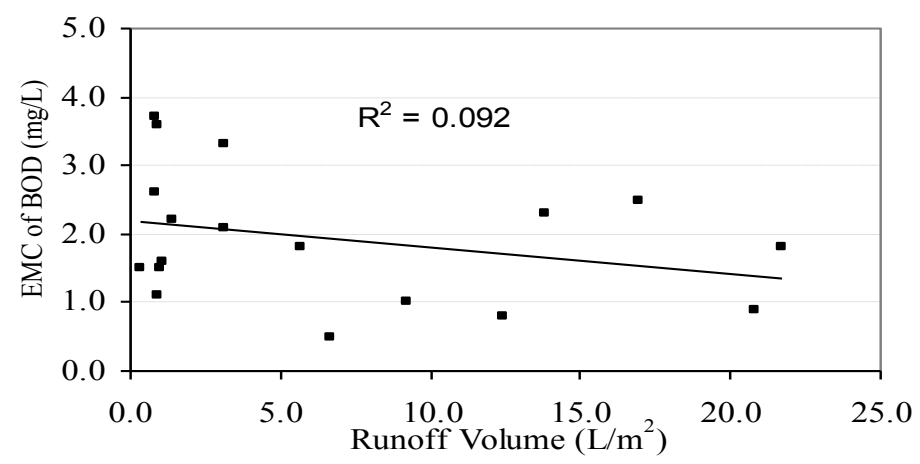

Figure 18.3 Correlation between the EMC of BOD and runoff volume.

To examine the interdependence among different pollutants, the correlation coefficients between the EMCs of two pollutants were estimated. These results indicate that several pollutants can be positively or negatively correlated with other pollutants, and several other pollutants are apparently correlated with each other. Among the calculated results, the highest coefficient, with a value of 0.84 , is the correlation between the EMCs of TP and nickel. While the coefficient is 0.79 between suspended solids (SS) and BOD, and 0.78 between SS and manganese, correlations between SS and other pollutants are generally weak. In particular, the EMCs of BOD and COD are negatively correlated with the correlation coefficient of 0.22 . The correlation between the EMCs of COD and BOD is illustrated in Figure 18.4, whereas in Figure 18.5, the $R^{2}$ value for the linear regression between the EMCs of iron and zinc is 0.62 . By comparison, the EMCs of other metals are generally not correlated to each other.

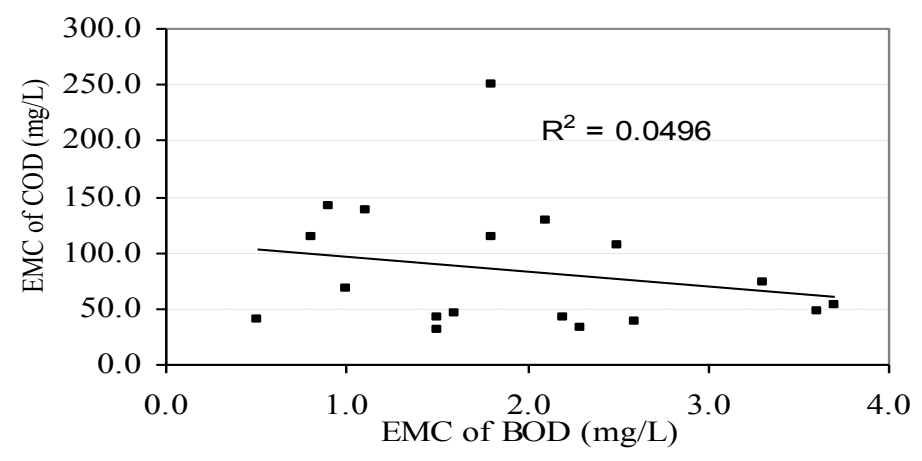

Figure 18.4 Correlation between the EMCs of COD and BOD. 


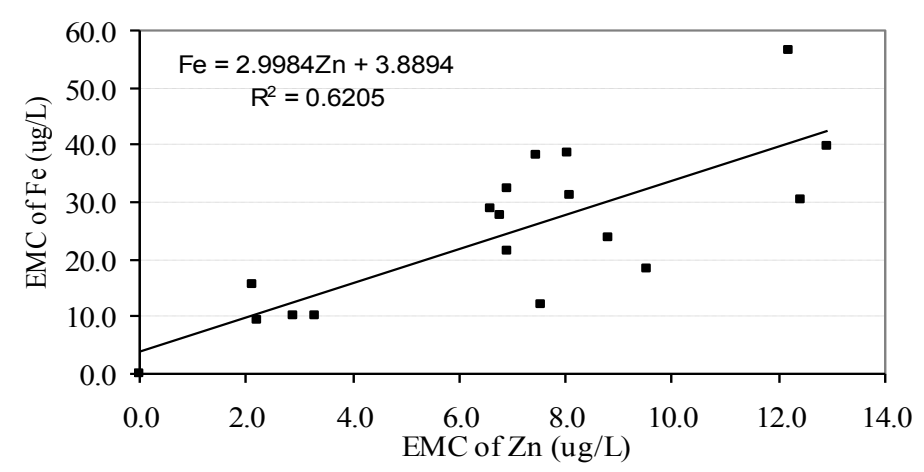

Figure 18.5 Correlation between the EMCs of iron and zinc.

The results from the correlation analyses indicate that there is no apparent dependence between pollutant EMCs and rainfall volume or intensity. Likewise, pollutant EMCs and runoff volume are poorly correlated. Without a strong correlation, the estimation of the EMCs from the rainfall characteristics or runoff volumes may be considered to be unreliable. Further correlation analysis on the interdependence between the EMCs of any two pollutants reveals that the EMCs for most pollutants do not appear to be highly correlated. A weak correlation between the EMCs of two pollutants may indicate that the EMC of a pollutant should not be considered as a surrogate to predict the EMC of another different pollutant.

\subsubsection{Correlation Analysis of Pollutant Loads}

Similar to the EMCs, the pollutant loads for the twelve water quality parameters in green roof stormwater runoff were analyzed for their interdependence. The unit area loadings (UAL) for each water quality variable are calculated as:

$$
U A L=\sum_{i=1}^{n} V_{i} \cdot E M C_{i} / A
$$

where:

$$
A=\text { catchment area from which runoff was sampled }\left(\mathrm{m}^{2}\right) \text {. }
$$

Table 18.2 shows a summary of the general statistics of green roof pollutant loads calculated from available measurements. The values in the table indicate that pollutant loads vary considerably between events, especially for the loads of COD and metals such as iron, copper and aluminum. The COD loads range from $10.0 \mathrm{mg} / \mathrm{m}^{2}$ to $2950 \mathrm{mg} / \mathrm{m}^{2}$, iron loads range from 3.0 $\mu \mathrm{g} / \mathrm{m}^{2}$ to $835 \mu \mathrm{g} / \mathrm{m}^{2}$, copper loads range from $32.4 \mu \mathrm{g} / \mathrm{m}^{2}$ to $1360 \mu \mathrm{g} / \mathrm{m}^{2}$ and 
aluminum loads range from $28.9 \mu \mathrm{g} / \mathrm{m}^{2}$ to $1147 \mu \mathrm{g} / \mathrm{m}^{2}$. The variation of pollutant loads is also reflected in their means and standard deviations. By comparison, the coefficients of skewness generally range from 1.03 to 3.52 for all the pollutants.

Table 18.2 General statistics for green roof pollutant loads.

\begin{tabular}{lcccc}
\hline \multicolumn{1}{c}{ Pollutant } & Range & Mean & $\begin{array}{c}\text { Standard } \\
\text { Deviation }\end{array}$ & Skewness \\
\hline $\mathrm{SS}(\mathrm{mg} / \mathrm{m} 2)$ & $0.4-54.1$ & 11.2 & 13.5 & 2.13 \\
$\mathrm{BOD}(\mathrm{mg} / \mathrm{m} 2)$ & $0.5-42.3$ & 11.0 & 13.3 & 1.58 \\
$\mathrm{COD}(\mathrm{mg} / \mathrm{m} 2)$ & $10.0-2950$ & 694 & 921.3 & 1.44 \\
$\mathrm{TP}(\mathrm{mg} / \mathrm{m} 2)$ & $0.02-14.0$ & 3.7 & 4.8 & 1.03 \\
$\mathrm{TKN}(\mathrm{mg} / \mathrm{m} 2)$ & $0.1-49.7$ & 11.1 & 15.4 & 1.59 \\
$\mathrm{Fe}(\mu \mathrm{g} / \mathrm{m} 2)$ & $3.0-835$ & 227 & 276.0 & 1.04 \\
$\mathrm{Cu}(\mu \mathrm{g} / \mathrm{m} 2)$ & $32.4-1360$ & 284 & 362.5 & 2.13 \\
$\mathrm{Al}(\mu \mathrm{g} / \mathrm{m} 2)$ & $28.9-1147$ & 292 & 338.8 & 1.33 \\
$\mathrm{Mn}(\mu \mathrm{g} / \mathrm{m} 2)$ & $0.4-43.3$ & 9.5 & 12.5 & 1.83 \\
$\mathrm{Ni}(\mu \mathrm{g} / \mathrm{m} 2)$ & $0.2-50.8$ & 12.2 & 16.2 & 1.39 \\
$\mathrm{Zn}(\mu \mathrm{g} / \mathrm{m} 2)$ & $0.7-218$ & 56.6 & 68.2 & 1.29 \\
$\mathrm{~Pb}(\mu \mathrm{g} / \mathrm{m} 2)$ & $0.8-249$ & 30.2 & 58.0 & 3.52 \\
\hline
\end{tabular}

The correlations between pollutant loads and rainfall intensity were calculated in order to determine the degree of dependence of the pollutant loads on rainfall intensity. It was found that the correlation coefficients are generally $<0.53$. The TP and nickel loads are found to be negatively correlated with rainfall intensity. Based on the calculated correlation coefficients for various pollutants, it is found that pollutant loads are not dependent on rainfall intensity. Taking COD load for example, in terms of the $R^{2}$ value of 0.01 as shown in Figure 18.6, the COD load is poorly correlated with rainfall intensity.

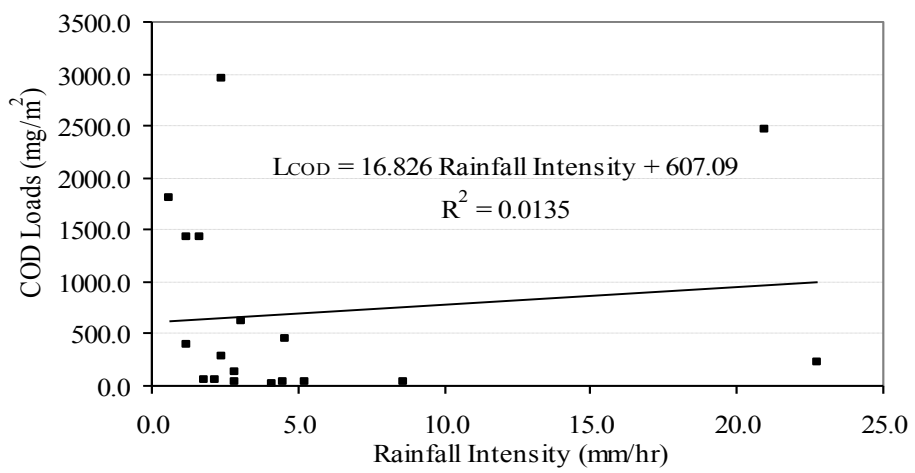

Figure 18.6 Correlation between COD load and rainfall intensity. 
For further comparison, the correlations between pollutant loads and rainfall volumes were also evaluated for all the pollutants. The calculated results show that the correlation coefficients generally range from 0.255 to 0.873 . The highest value of 0.873 is the coefficient for the correlation between copper load and rainfall volume, and the lowest value of 0.255 is the correlation coefficient between SS load and rainfall volumes. No pollutant loads are found to be negatively correlated with rainfall volumes. While somewhat better than previous results, the correlations between pollutant loads and rainfall volumes are not clearly defined for most pollutants. Figure 18.7 shows the correlation between the COD load and rainfall volumes (the $R^{2}$ value is 0.1557 ).

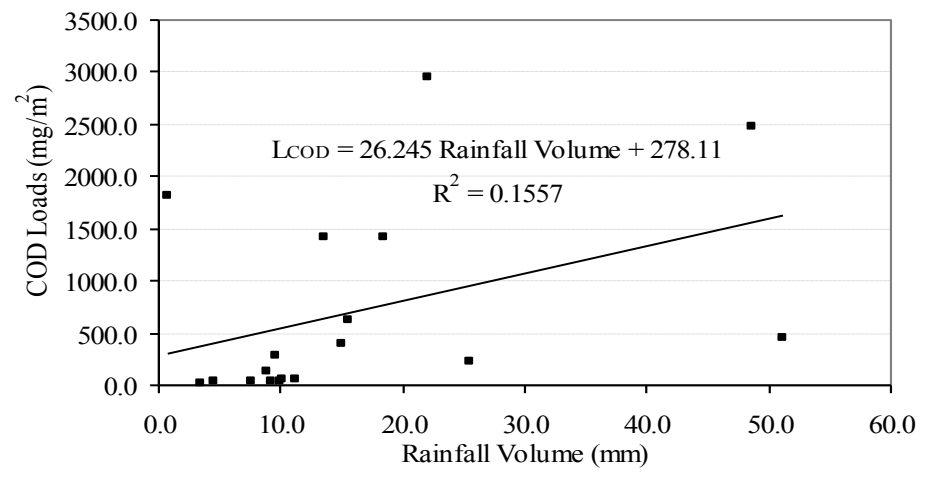

Figure 18.7 Correlation between COD load and rainfall volume.

In addition to rainfall volumes and intensities, the correlations between pollutant loads and runoff volumes were also estimated. It is found that pollutant loads are highly correlated with runoff volumes. The calculated results of the correlation coefficients range from 0.73 to 0.982 . The lowest value of 0.73 is the coefficient for the correlation between lead load and runoff volume, and the highest value of 0.982 is the coefficient for the correlation between iron load and runoff volume. No pollutant loads are found to be negatively correlated with runoff volume. Figure 18.8 shows the correlation between COD load and runoff volume with the $R^{2}$ value of 0.82 . Figures 18.9 to 18.11 show the correlations between TP load and rainfall intensity, rainfall volume and runoff volume, respectively. As indicated in the figures, TP load is strongly correlated with runoff volume with the $R^{2}=0.90$. In comparison, the rainfall volume or intensity is poorly correlated with TP load. 


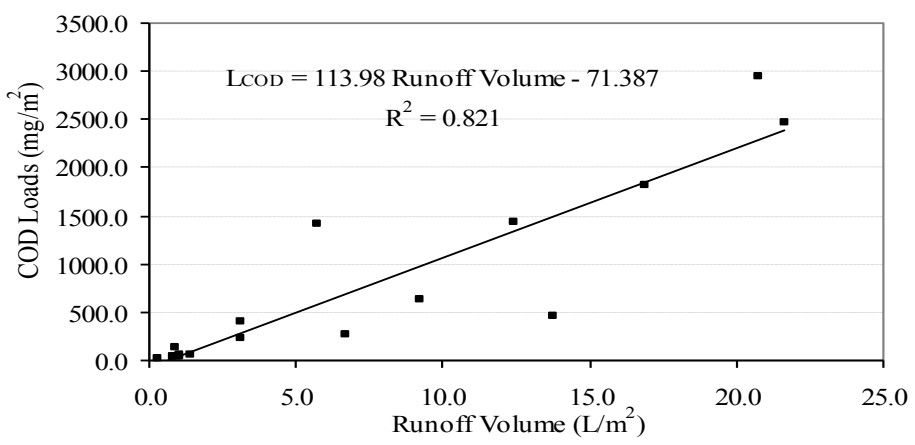

Figure 18.8 Correlation between COD load and runoff volume.

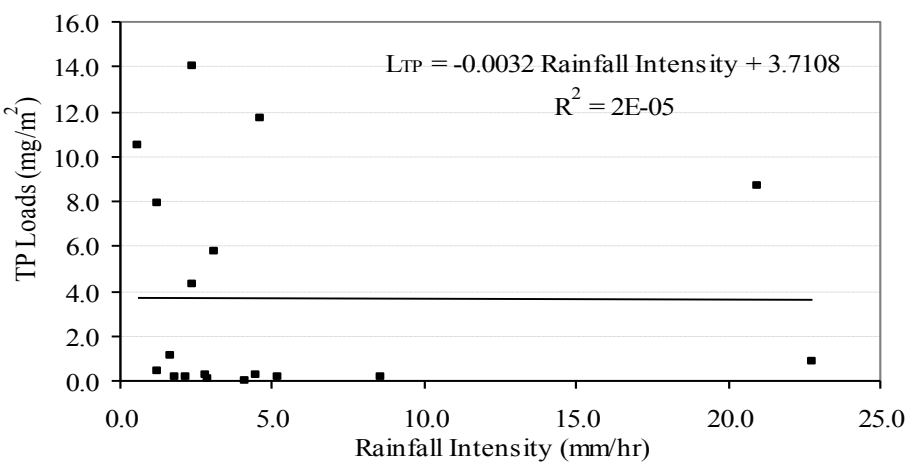

Figure 18.9 Correlation between TP load and rainfall intensity.

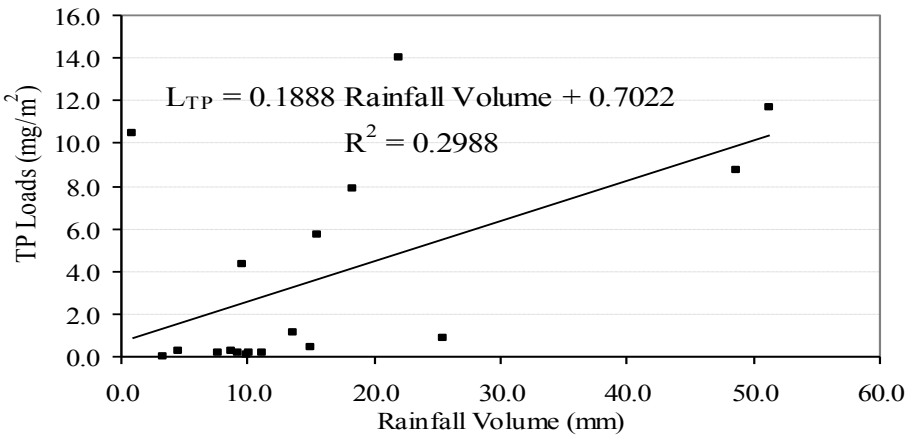

Figure 18.10 Correlation between TP load and rainfall volume. 


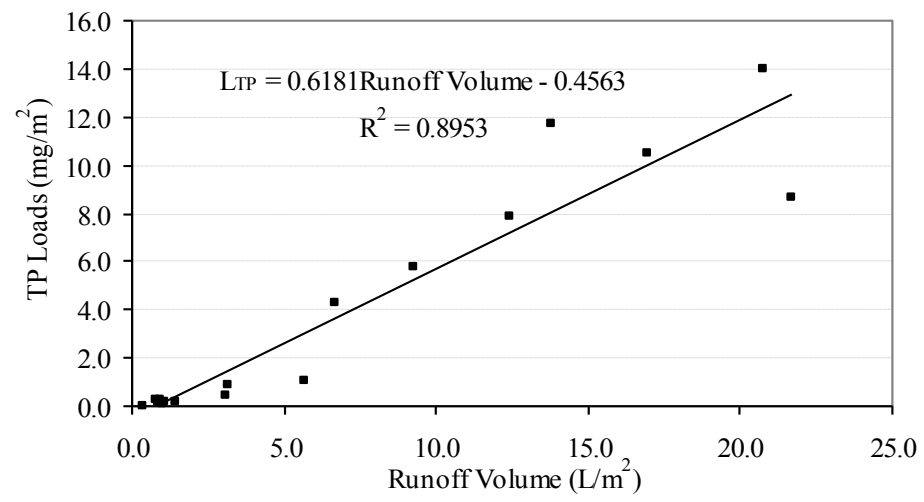

Figure 18.11 Correlation between TP load and runoff volume.

As shown earlier, the correlation between the EMCs of two pollutants is generally weak. In contrast, the loads of two pollutants are found to be well correlated. The correlation coefficients between SS load and other pollutants range from 0.60 to 0.94 (Table 18.3 ). The SS load is highly correlated with the manganese load, with the correlation coefficient of 0.94 . The correlation coefficients between the zinc load and other pollutants range from 0.64 to 0.97. There is a strong correlation between the loads of zinc and iron with a correlation coefficient of 0.97 . The correlation coefficients between the load of iron and other pollutants generally range from 0.75 to 0.98 . In addition, the load of aluminum is also highly correlated with other pollutant loads.

The strong correlation between two paired pollutant loads makes it possible to consider one pollutant as a surrogate to estimate the other pollutant load, perhaps when measurements of the pollutant are missing or unreliable. In other cases it may be necessary to interpolate water quality data between two measurements or extend the data by extrapolation of existing loads.

Table 18.3 Correlation coefficients of pollutant loads.

\begin{tabular}{cccc}
\hline Pollutant & $\mathrm{SS}(\mathrm{mg} / \mathrm{m} 2)$ & $\mathrm{Fe}(\mu \mathrm{g} / \mathrm{m} 2)$ & $\mathrm{Zn}(\mu \mathrm{g} / \mathrm{m} 2)$ \\
\hline $\mathrm{SS}(\mathrm{mg} / \mathrm{m} 2)$ & - & 0.845 & 0.939 \\
$\mathrm{BOD}(\mathrm{mg} / \mathrm{m} 2)$ & 0.896 & 0.911 & 0.917 \\
$\mathrm{COD}(\mathrm{mg} / \mathrm{m} 2)$ & 0.740 & 0.898 & 0.895 \\
$\mathrm{TP}(\mathrm{mg} / \mathrm{m} 2)$ & 0.791 & 0.907 & 0.890 \\
$\mathrm{TKN}(\mathrm{mg} / \mathrm{m} 2)$ & 0.857 & 0.955 & 0.928 \\
$\mathrm{Fe}(\mu \mathrm{g} / \mathrm{m} 2)$ & 0.845 & - & 0.967 \\
$\mathrm{Cu}(\mu \mathrm{g} / \mathrm{m} 2)$ & 0.602 & 0.859 & 0.745 \\
$\mathrm{Al}(\mu \mathrm{g} / \mathrm{m} 2)$ & 0.855 & 0.978 & 0.947 \\
$\mathrm{Mn}(\mu \mathrm{g} / \mathrm{m} 2)$ & 0.939 & 0.900 & 0.930 \\
$\mathrm{Ni}(\mu \mathrm{g} / \mathrm{m} 2)$ & 0.745 & 0.805 & 0.807 \\
$\mathrm{Zn}(\mu \mathrm{g} / \mathrm{m} 2)$ & 0.939 & 0.967 & - \\
$\mathrm{Pb}(\mu \mathrm{g} / \mathrm{m} 2)$ & 0.648 & 0.747 & 0.642 \\
\hline
\end{tabular}




\subsection{Probability Distributions of Pollutant Loads}

\subsubsection{Cumulative Distribution Function of Pollutant Loads}

The cumulative distribution function (CDF) of a pollutant load is defined as the probability that a series of pollutant load data has a value less than the given value; whereas the empirical distribution function of a pollutant load is a cumulative probability distribution function that concentrates probability $(1 / n)$ at each of the $n$ measurements in a sample. The standard normal version of the function calculates the probability for a series with mean 0 and standard deviation 1 . The CDF plots are extremely useful for finding the fluorescence of a distribution corresponding to any given percentile. The sample plot of pollutant load indicates the median (50th percentile), 75th, and 90th percentiles for the sample data.

To identify the statistical behavior of pollutant loads, probability distributions were fitted to the observed data. For this purpose, normal, log-normal and exponential distributions were selected in this study. Figure 18.12 shows a comparison of the CDFs of COD load in terms of the three selected probability distributions. As indicated in the figure, the CDF of COD load from the log-normal distribution matches the observed distribution reasonably well. The normal distribution tends to significantly overestimate the CDF of COD load, whereas the exponential distribution appears to provide estimates somewhere in between the two for those events with a medium range load. A comparison of the CDF of chloride load in terms of the three selected probability distributions is shown in Figure 18.13. With reference to the empirical CDF of chloride load, the best fit to the observed data is the log-normal distribution. Both the normal and exponential distributions are likely to overestimate the CDFs of chloride load.

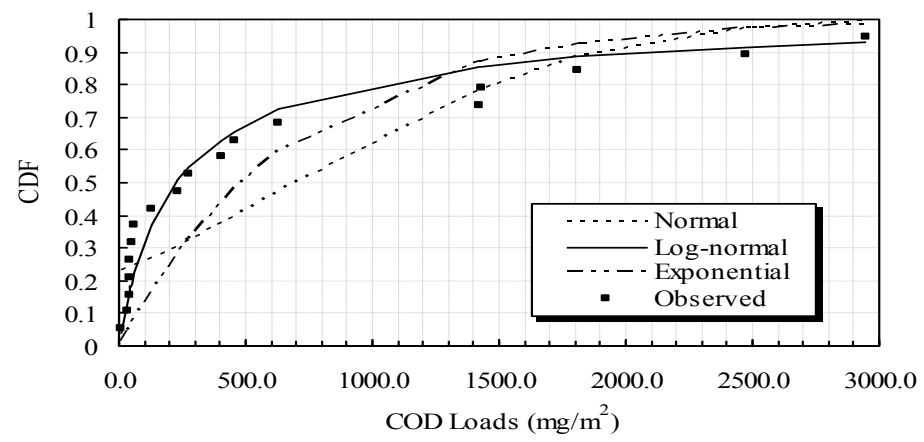

Figure 18.12 Comparison of the CDFs of COD load. 


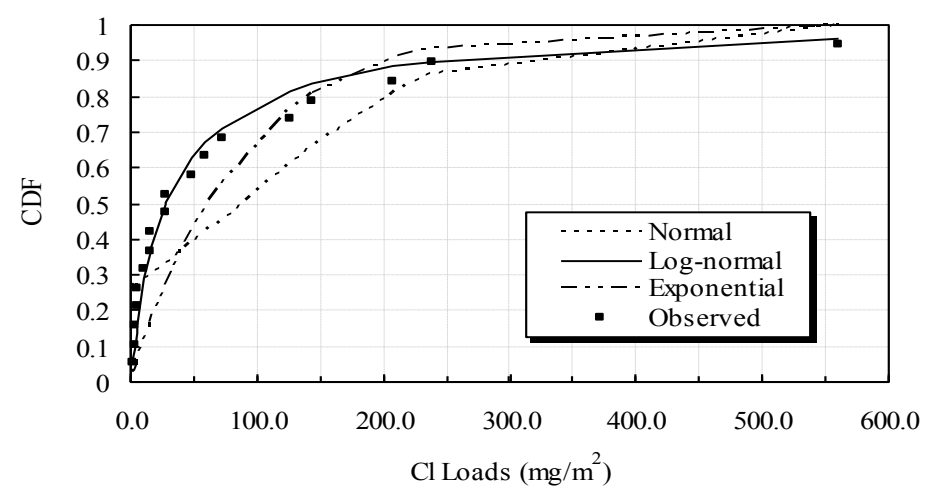

Figure 18.13 Comparison of the CDFs of chloride load.

For lead load, Figure 18.14 shows that the log-normal distribution fits the observed data better than either the normal or the exponential distributions. Similarly to COD load in Figure 18.12 and chloride load in Figure 18.13, the normal distribution is more likely to overestimate the CDF of lead load for those events with a medium range of lead load. By comparison, the estimates from the exponential distribution are more close to the estimates of the lognormal distribution. As for SS load, log-normal distribution is still a better choice than the other two distributions as indicated in Figure 18.15. Similar patterns are also observed from the plots of the CDFs for other pollutant loads.

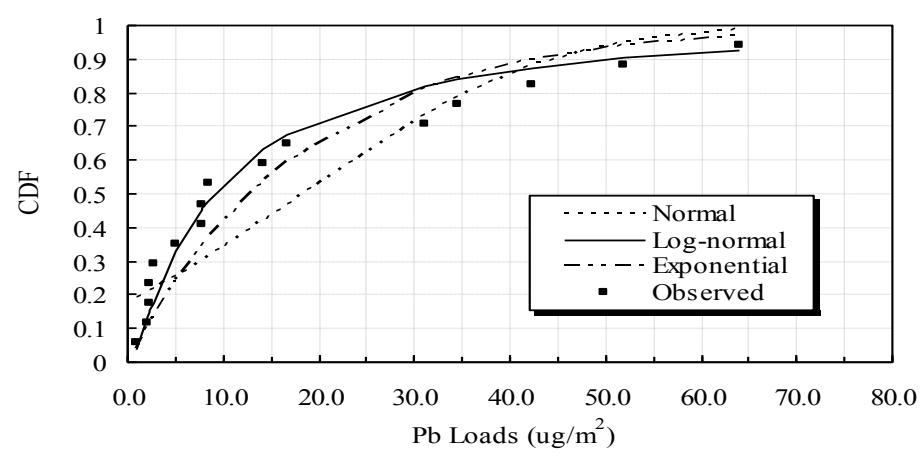

Figure 18.14 Comparison of the CDFs of lead load. 


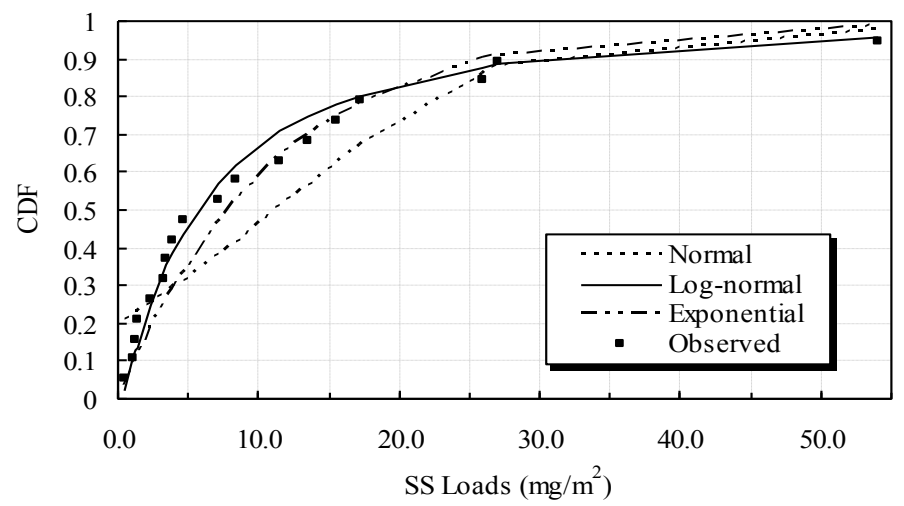

Figure 18.15 Comparison of the CDFs of SS load.

In summary, for most pollutants found in garden runoff, the log-normal distribution fits the sampled data of pollutant load reasonably well. The shape of the CDF based on the normal distribution appears to be oversimplified and deviates from the empirical CDF of pollutant load. Considering the deviations between the empirical and normal CDFs, the normal distribution tends to underestimate the values of the CDF of pollutant load. By comparison, the exponential distribution is likely to provide estimates in between the two other distributions for those events with a medium range load.

\subsubsection{Comparison of the Observed and Estimated Pollutant Loads}

It is of interest to examine whether suspended solids can be considered as a surrogate to predict other pollutant loads since it is a commonly measured water quality parameter. For this purpose, the degree of dependence of other pollutants on suspended solids is further examined in terms of pollutant loads. Figure 18.16 shows the correlation plot of SS load against TKN load with $R^{2}=0.73$. Based on the regression equation shown in the figure, the TKN load is calculated with inputs of the SS load, and the CDFs of the predicted TKN load were calculated and plotted against the observed TKN load along with the fitted CDF of TKN load (log-normal distribution) in Figure 18.17. The figure shows that using SS load as a surrogate is likely to overestimate TKN load for those events with small and medium quantity loads. As a comparison, there may be other pollutants which can be used as a better surrogate than the SS load for predicting other pollutant loads. Therefore, additional analysis was performed for pollutants such as iron. 


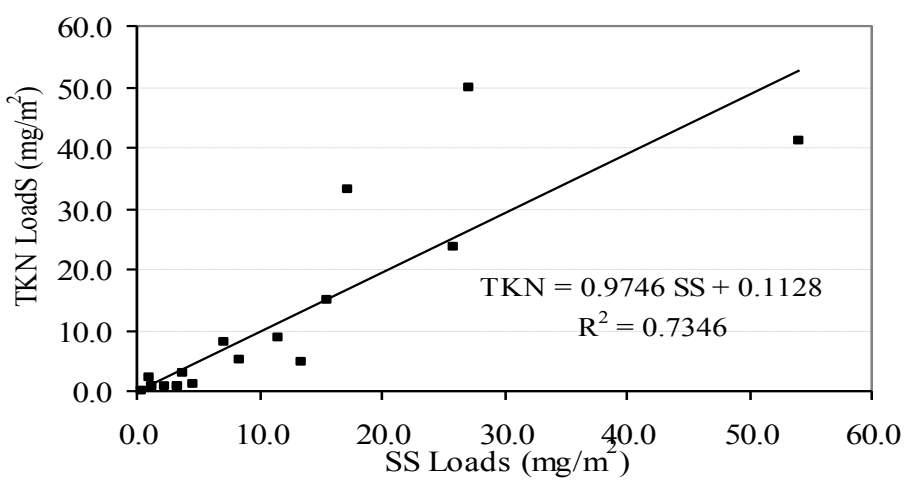

Figure 18.16 Correlation between SS and TKN load.

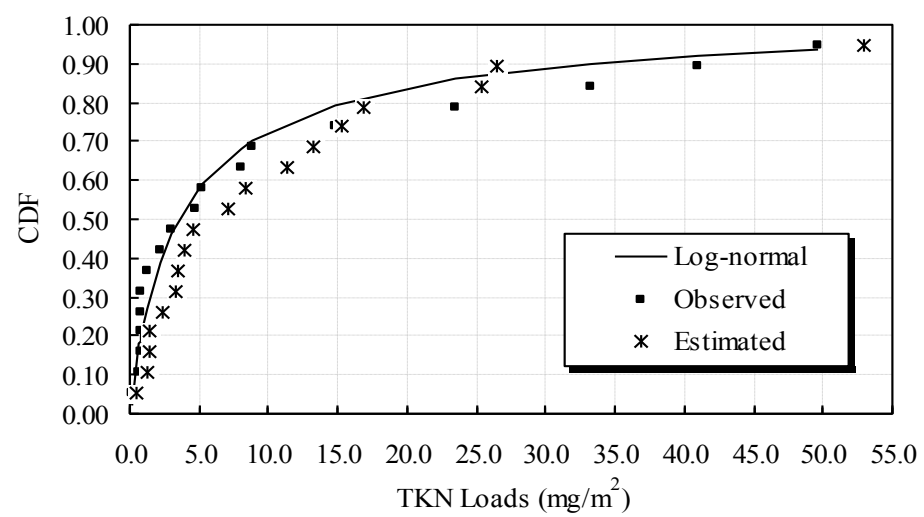

Figure 18.17 Comparison of the observed and the estimated TKN load.

To further verify the possibility of using alternative pollutants such as iron instead of SS as a surrogate for predicting the loads of other pollutants (e.g. COD), SS load is plotted against COD load in Figure 18.18 with $R^{2}=0.55$, whereas iron load is plotted against COD load in Figure 18.19 with $R^{2}=0.81$. The correlation between iron load and COD load is stronger than that between SS load and COD load. Based on the regression equation in Figure 18.19, the COD load is estimated with the inputs of the iron load, and the CDF of the estimated COD load was calculated and plotted against the empirical COD load along with the fitted CDF of the COD load (lognormal distribution) in Figure 18.20. In comparison, the CDF of the estimated COD load was also calculated with the regression equation in Figure 18.18 , in which the SS load is used as a surrogate. The estimated CDF of the COD load is also plotted in Figure 18.20. The estimated CDF of the COD 
load with the iron load as a surrogate matches the observed load and the fitted distribution well for those events of relatively low load. Using the SS load as a surrogate is likely to underestimate the CDF of the COD load.

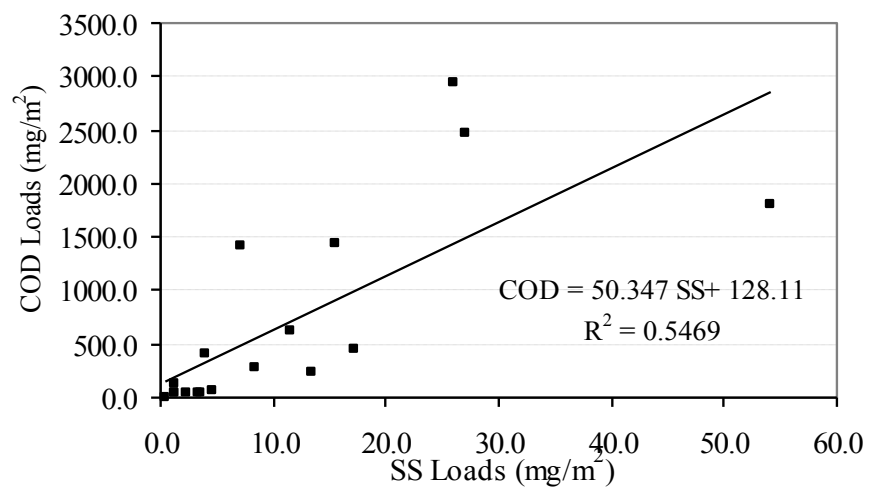

Figure 18.18 Correlation between SS load and COD load.

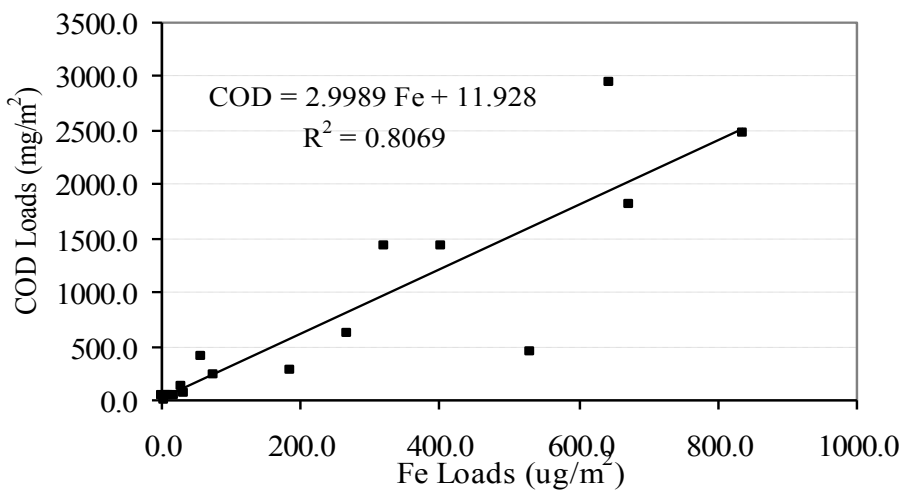

Figure 18.19 Correlation between iron load and COD load.

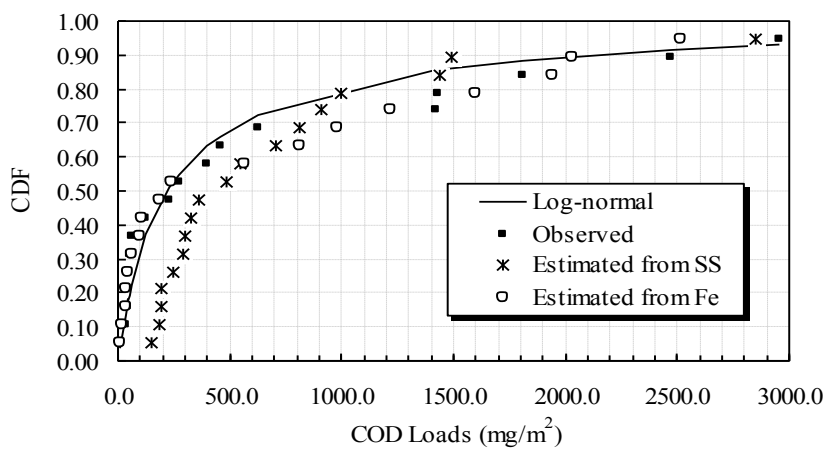

Figure 18.20 Comparison of the observed load and the estimated COD load. 
However, additional analysis shows that the SS load can be used as a good surrogate to predict the manganese and zinc loads.

\subsubsection{Exceedance Probability of Pollutant Loads}

As shown earlier, the CDF based on the log-normal distribution fits the observed pollutant loads reasonably well. Therefore, the sample of observed pollutant loads may be assumed to be log-normally distributed rather than normally or exponentially distributed. To investigate the statistical outlook of pollutant loads, the exceedance probabilities of pollutant loads were extrapolated based on sample statistics (mean, deviation and skewness). Since pollutant loads are assumed to be log-normally distributed, the return periods of pollutant loads can be estimated with a log-normal distribution, which may serve as a reference datum to determine the reliability of pollutant loads estimated from other probability distributions, such as Pearson or logPearson.

Figure 18.21 shows a comparison of the exceedance probability of COD load from the four selected probability distributions (normal, log-normal, Pearson and log-Pearson). The plot from the results of the log-Pearson distribution almost overlaps the plot of the log-normal distribution as shown in the figure, so it is reasonable to assume that the sample of COD loads can also be fitted by the log-Pearson distribution reasonably well, in addition to the log-normal distribution. By comparison, estimates from both the Pearson and normal distributions significantly deviate from estimates of the lognormal or log-Pearson distributions. The normal or Pearson distributions are likely to significantly underestimate the return period of COD loads, especially for large COD loading events. For example, the $100 \mathrm{y}$ COD load is about $12820 \mathrm{mg} / \mathrm{m}^{2}$ for the log-normal distribution, $12410 \mathrm{mg} / \mathrm{m}^{2}$ for the log-Pearson distribution, but $2840 \mathrm{mg} / \mathrm{m}^{2}$ for the normal distribution and $3730 \mathrm{mg} / \mathrm{m}^{2}$ for the Pearson distribution.

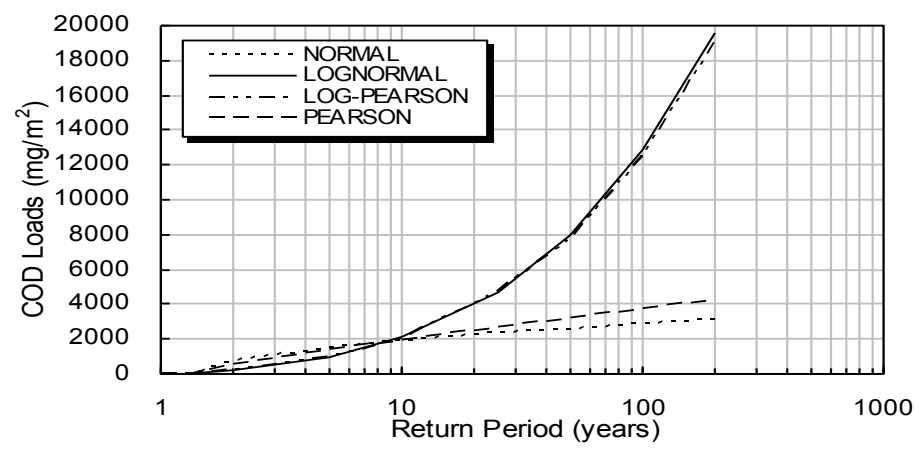

Figure 18.21 Exceedance probability distribution of COD loads. 
To evaluate the probability distribution of aluminum loads, the exceedance probabilities were calculated with the statistics estimated from the sample of aluminum load and plotted according to different probability distributions in Figure 18.22. The figure shows that the log-Pearson distribution is much closer to the log-normal distribution than either the normal or the Pearson distributions. As a result, estimates from the normal and Pearson distributions significantly deviate from estimates of the log-normal or logPearson distributions for aluminum load for those events with a relatively high load. For example, the 50 y aluminum loading is about $2090 \mu \mathrm{g} / \mathrm{m}^{2}$ for the log-normal distribution, $2320 \mu \mathrm{g} / \mathrm{m}^{2}$ for the log-Pearson distribution, but $1010 \mathrm{mg} / \mathrm{m}^{2}$ for the normal and $1210 \mathrm{mg} / \mathrm{m}^{2}$ for the Pearson distribution.

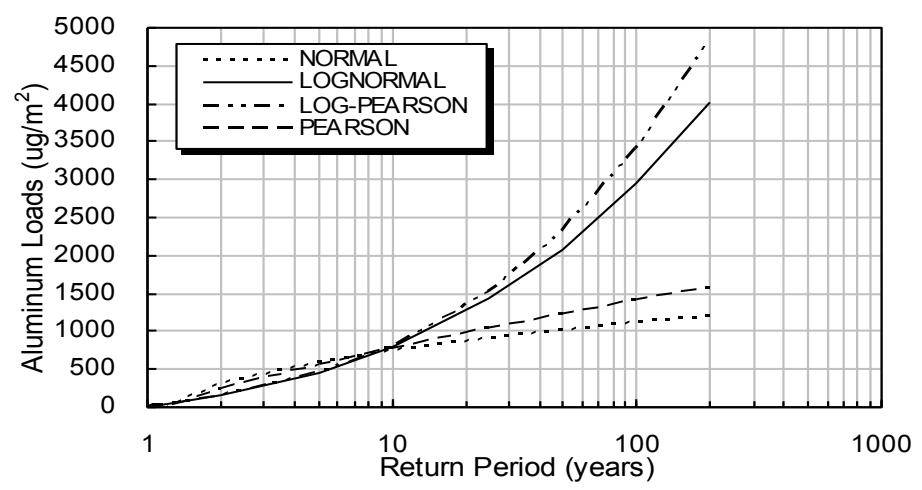

Figure 18.22 Exceedance probability distribution of aluminum load.

The plots of the exceedance probability distributions of calcium loads (Figure 18.23) and TKN load (Figure 18.24) also show similar patterns. In both figures, the log-Pearson distribution is capable of providing estimates of pollutant load that are close to the estimates of the log-normal distribution. By comparison, estimates from the normal and Pearson distributions are significantly different.

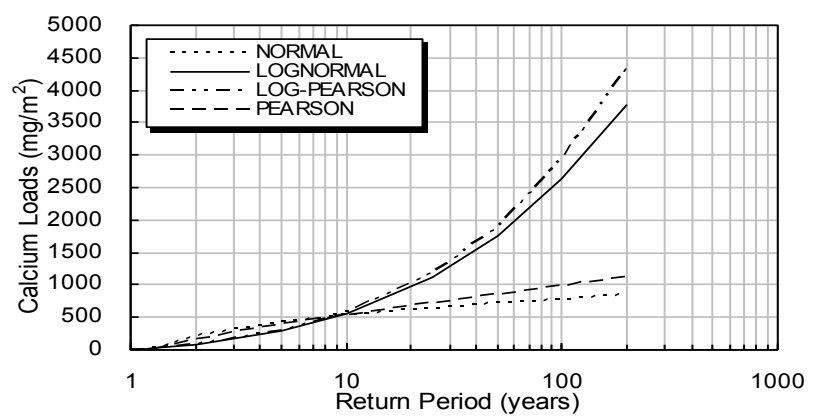

Figure 18.23 Exceedance probability distributions of calcium load. 


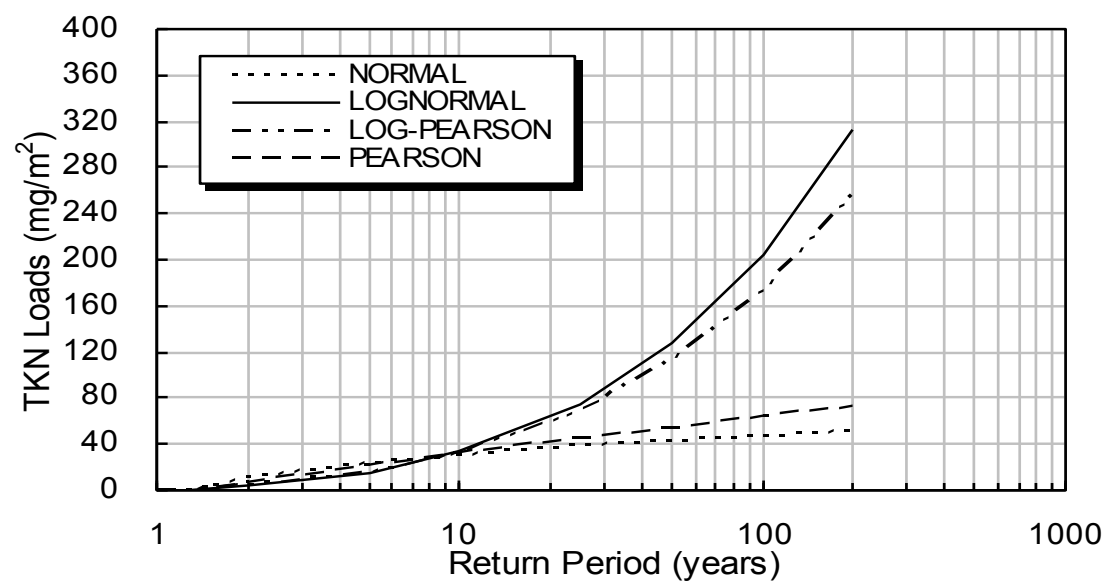

Figure 18.24 Exceedance probability distribution of TKN load.

\subsubsection{Summary of Results}

A summary of the qualitative correlation results between two pollutants is given in Table 18.4, whereas Table 18.5 shows a summary of the $R$ squared values for each pollutant used as a surrogate indicator.

Table 18.4 Qualitative correlation results between two pollutant loads.

\begin{tabular}{|c|c|c|c|c|c|c|c|c|c|c|c|}
\hline & SS & BOD & $\mathrm{COD}$ & TP & TKN & $\mathrm{Fe}$ & $\mathrm{Cu}$ & $\mathrm{Al}$ & $\mathrm{Mn}$ & $\mathrm{Ni}$ & $\mathrm{Zn}$ \\
\hline $\mathrm{Pb}$ & $\mathrm{D}$ & $\mathrm{D}$ & $\mathrm{D}$ & $\mathrm{E}$ & $\mathrm{C}$ & $\mathrm{C}$ & $\mathrm{C}$ & $\mathrm{C}$ & $\mathrm{D}$ & $\mathrm{E}$ & $\mathrm{D}$ \\
\hline $\mathrm{Zn}$ & B & B & B & B & B & A & C & B & B & C & \\
\hline $\mathrm{Ni}$ & $\mathrm{C}$ & $\mathrm{C}$ & D & B & $\mathrm{C}$ & $\mathrm{C}$ & D & D & D & & \\
\hline $\mathrm{Mn}$ & B & A & C & C & A & B & B & B & & & \\
\hline $\mathrm{Al}$ & B & B & C & B & A & A & B & & & & \\
\hline $\mathrm{Cu}$ & D & B & D & C & B & B & & & & & \\
\hline $\mathrm{Fe}$ & C & B & B & B & A & & & & & & \\
\hline TKN & B & A & C & C & & & & & & & \\
\hline TP & C & C & C & & & & & & & & \\
\hline COD & C & C & & & & & & & & & \\
\hline BOD & B & & & & & & & & & & \\
\hline
\end{tabular}

A: Strongly correlated (correlation coefficient $\geq 0.95$ )

B: Well correlated $(0.85 \leq$ correlation coefficient $<0.95)$

C: Correlated $(0.70 \leq$ correlation coefficient $<0.85)$

D: Somewhat correlated $(0.55 \leq$ correlation coefficient $<0.70)$

E: Poorly correlated (correlation coefficient $<0.55$ ). 
Table $18.5 R$-squared values of a pollutant load used as a surrogate indicator for estimation of another pollutant load.

\begin{tabular}{lcccccccccrr}
\hline & SS & BOD & COD & TP & TKN & Fe & Cu & Al & Mn & Ni & Zn \\
\hline $\mathrm{Pb}$ & 0.42 & 0.31 & 0.39 & 0.67 & 0.32 & 0.48 & 0.19 & 0.46 & 0.37 & 0.69 & 0.50 \\
$\mathrm{Zn}$ & 0.88 & 0.84 & 0.80 & 0.79 & 0.86 & 0.94 & 0.56 & 0.90 & 0.86 & 0.65 & \\
$\mathrm{Ni}$ & 0.56 & 0.58 & 0.40 & 0.89 & 0.57 & 0.65 & 0.40 & 0.58 & 0.52 & & \\
$\mathrm{Mn}$ & 0.88 & 0.95 & 0.51 & 0.60 & 0.92 & 0.81 & 0.63 & 0.88 & & & \\
$\mathrm{Al}$ & 0.73 & 0.87 & 0.70 & 0.78 & 0.95 & 0.96 & 0.79 & & & & \\
$\mathrm{Cu}$ & 0.36 & 0.75 & 0.43 & 0.52 & 0.83 & 0.74 & & & & & \\
$\mathrm{Fe}$ & 0.71 & 0.83 & 0.81 & 0.82 & 0.91 & & & & & & \\
$\mathrm{TKN}$ & 0.74 & 0.96 & 0.59 & 0.70 & & & & & & & \\
$\mathrm{TP}$ & 0.63 & 0.63 & 0.62 & & & & & & & & \\
$\mathrm{COD}$ & 0.55 & 0.50 & & & & & & & & & \\
$\mathrm{BOD}$ & 0.80 & & & & & & & & & \\
\hline
\end{tabular}

\subsection{Conclusions}

For the characterization of green roof stormwater runoff quality, it is found that the EMCs are not correlated with rainfall volume or intensity. The correlation between the EMCs and rainfall volumes are also weak. In comparison, a better correlation is observed between pollutant load and runoff volume. The correlation between two paired pollutant loads is stronger than the correlation between two paired EMCs. The results from correlation analysis also show that metals such as iron, zinc or aluminum may be considered as better surrogates than the SS for predicting other pollutant loads. For several commonly used probability distributions, it is found that the CDF of the log-normal distribution can fit the observed pollutant loads reasonably well, whereas normal and exponential distributions are likely to overestimate the CDFs of pollutant loads. Based on sample statistics and with reference to estimates from the log-normal distribution, the extrapolation of the exceedance probability of pollutant loads shows that the log-Pearson distribution can provide close estimates for most of other pollutants. Normal and Pearson distributions appear to differ significantly from the log-normal and logPearson estimates for a given return period. The data used for this study was still limited for conducting more in-depth analyses including the investigation of seasonal variations in stormwater quality. It is strongly recommended that more updated monitoring data should be used in the future study.

\section{References}

Bliss, D. J., Neufeld, R. D. and Riles, R. J. 2009. Storm Water Runoff Mitigation Using a Green Roof. Environmental engineering science, 26 (2), 407-417. 
Canadian Council of Ministers of the Environment. 1999. Canadian Environmental Quality Guidelines, Canadian Council of Ministers of the Environment, Winnipeg.

Flint, K. R. and Davis, A. P. 2007. Pollutant mass flushing characterization of highway stormwater runoff from an ultra-urban area. J. Envir. Engrg., ASCE, 133(6), 616-26.

Johnston, J. and Newton, J. 1996. Building Green: A Guide for Using Plants on Roofs, Walls and Pavements. The London Ecology Unit, London.

Keiko, W. and Shigeo, F. 2006. Characterization of stormwater runoff processes from urban roadways. Journal of Japan Society on Water Environment, 29(11), 699-704.

Lee, J. H. and Bang, K. W. 2000. Characterization of urban stormwater runoff. Water research, 34(6), 1773-80.

McLeod, S. M., Kells, J. A., and Putz, G. J. 2006. Urban runoff quality characterization and load estimation in Saskatoon, Canada. J. Envir. Engrg., ASCE, 132(11), 147081.

Seters, T. V., Rocha, L., Smith, D. and MacMillan, G. 2009. Evaluation of green roofs for runoff retention, runoff quality, and leachability. Water Quality Research Journal of Canada, 44(1): 33-47.

Teemusk, A. and Mander, Ülo 2007. Rainwater runoff quantity and quality performance from a greenroof: The effects of short-term events. Ecological Engineering, 30(3): 271-277.

Toronto and Region Conservation Authority (TRCA), 2006. Evaluation of an Extensive Greenroof York University, Toronto, Ontario

USEPA, 2009. Green Roofs for Stormwater Runoff Control. Office of Research and Development, National Risk Management Research Laboratory - Water Supply and Water Resources Division. EPA/600/R-09/026, February.

Van Buren, M.A., Watt, W.E. and Marsalek, J. 1997. Application of the log-normal and normal distributions to stormwater quality parameters. Water Research, 31(1): 95104. 
